


\title{
On the Duffin-Kemmer-Petiau Algebra and the Generalized Phase Space
}

\author{
Marco Cezar B. Fernandes ${ }^{(1)}$ and J. David M. Vianna ${ }^{(1,2)}$ \\ ${ }^{(1)}$ Núcleo de Física Atômica, Molecular e Fluidos \\ Instituto de Física, Universidade de Brasília \\ 70910-900 Brasília, DF, Brazil \\ ${ }^{(2)}$ Instituto de Física, Universidade Federal da Bahia \\ Campus Universitário de Ondina \\ 40210-340 Salvador, BA, Brazil
}

Received 9 September, 1998

\begin{abstract}
The Duffin-Kemmer-Petiau (DKP) relativistic equation has been recently uded to study the interactions of spinless mesons with nuclei. In view of this interest and also the interest to determine the phase space picture for hadronic quantum theory of the DKP equation on the generalized phase space proposed by Bohm and Hiley. Our development is based on the algebraic calculus introduced by Schönberg and uses the idea of algebraic spinors due to Riesz and Cartan. The free DKP particle and the more general case of the DKP particle in a prescribed external electromagnetic field are considered $\Gamma$ and we obtain the DKP Liouville type equations for these cases.
\end{abstract}

\section{Introduction}

The description of quantum systems using a generalized form of the Liouville equation is apropriate in the context where one wants to use a classical formalism to understand the nature of physical attributes usually associated with quantum mechanics. Quantities like spin $\Gamma$ for instance $\Gamma$ can be given a formulation in terms of a Liouville type equation in a relativistic phase space [1]. In such cases the spinorial character of the theory is contained essentially in the algebraic structure of the Liouville superoperator. Therefore $\mathrm{\Gamma it}$ is important to know how the spin algebras are combined with the differential operators involved in the Liouville equation as well as the domain of these operators. This combined structure is then what we mean by a generalized Liouville equation. In the relativistic case the role played by the so-called geometric algebras has been given attention as a way of understanding the nature of the relativistic phase space [1]. Following suggestions of Schönberg and Bohm[1][2] $\Gamma$ Holland [3] derived relativistic phase space equations for a massive spin $\frac{1}{2}$ particle in an external electromagnetic field. In his development $\Gamma$ Holland takes the complex Dirac algebra $C_{4}$ and obtains the phase space representation of the Dirac and Feynman-Gell-Mann equations.

Recently [ $4 \Gamma$ and Refs. cited therein] the firstorder relativistic Duffin-Kemmer-Petiau (D.K.P) equation has been analyzed as part of a program to study the interactions of spinless mesons with nuclei. Simultaneously (and independently) the D.K.P algebras which are associated to D.K.P equation have been studied from a modern perspective [5].

In view of this recent interest in the use of the D.K.P relativistic equation Tthe study of D.K.P algebras and also the interest to determine the phase space formulation for hadronic quantum theory [6] $\Gamma$ we propose in this paper a derivation of the phase space representation of the D.K.P equation for scalar particles. We will follow the algebraic calculus introduced by Schönberg[2] and the mathematical development of Bohm and Hiley [1] based on the idea of algebraic spinors [7]. In doing so $\Gamma$ we show that it is also possible to write relations of classical type in a relativistic phase space by means 
of the D.K.P. algebra. The paper is organized as follows. In section 2Гalong the lines of Schönberg [2] Гwe begin with the D.K.P equation for the algebra in the sense that the D.K.P operator acts on a subspace of the algebra. This allows us to suggest a broader meaning for this operator aiming to generalize the equation for further integer spins. This will also yields a relation with the Dirac algebraic spinors. We will then take from that and use a relativistic version of the WignerMoyal transformation to make the passage to a phase space equation. Section 3 will follow with an interpretation of our D.K.P Liouville type equation corresponding to scalar particles in an external electromagnetic field. Section 4 closes with some conclusion remarks.In the Appendix the notation and some formal definitions related to spinor spaces $\Gamma$ Grassmann $\Gamma$ Dirac and D.K.P. algebras are presented.

\section{Phase space formulation of the D.K.P relativistic equa- tion}

In this section $\Gamma$ following Schönberg [2] $\Gamma$ we begin with the D.K.P equation in the algebra in order to give a broader meaning to this relativistic equation in terms of the algebraic spinors [7]. This will allow us to use the techniques introduced in [1] further on in the paper.

The D.K.P operator in the algebra combines the covariant vector $\partial / \partial x^{\mu} \equiv \partial_{\mu}$ with the generator of the D.K.P algebra $\beta^{\mu}$. The evolution D.K.P operator is the contraction

$$
\partial_{\mu} \beta^{\mu}, \quad \mu=1,2,3,4
$$

The free D.K.P particle of mass $m$ is described by the following equation:

$$
\left(\partial_{\mu} \beta^{\mu}+m\right) \Psi=0
$$

This is an equation defining the eigenstates $\Psi$ of $\partial_{\mu} \beta^{\mu}$. This equation is being seen here as a generalization of the usual D.K.P equation [2] in the sense that the $\Psi$ is an element of the minimum left ideal of an extended Grassmann algebra. Following the Appendix $\Gamma$ this $\Psi$ takes the explicit form:

$$
\Psi=\sum_{q=0}^{n}(q !)^{-1} A_{k_{1} \ldots k_{q}}\left(P^{k_{1} \ldots k_{q}}\right), \quad \Psi \in \mathcal{C}_{\mathcal{P}}(\mathbf{W})
$$

Now from the general form

$$
\begin{aligned}
\beta_{\mu}^{(p)} & =\left(\Pi_{p}\right)\left(\mathbf{e}_{\mu}\right)+g_{\mu \nu}\left(\mathbf{e}^{\nu}\right)\left(\Pi_{p}\right) \\
& =\left(\mathbf{e}_{\mu}\right)\left(\Pi_{p+1}\right)+g_{\mu \nu}\left(\mathbf{e}^{\nu}\right)\left(\Pi_{p}\right)
\end{aligned}
$$

it is easy to see that the action of a $\beta_{\mu}^{(p)}$ on a spinor $\Psi$ projects it down to the space of antisymmetric tensors of order $p+1$ and $p$. This then shows the difference between the Dirac algebraic spinors and D.K.P spinors in this algebraic context.

In the present work $\Gamma$ we restrict our analysis to the scalar particles $\Gamma$ i.e we take $p=0$. Thus

$$
\begin{aligned}
\beta_{\mu}^{(0)}=\beta_{\mu} & =\left(\mathbf{e}_{\mu}\right)\left(\Pi_{1}\right)+g_{\mu \nu}\left(\mathbf{e}^{\nu}\right)\left(\Pi_{0}\right) \\
& =\left(\mathbf{e}_{\mu}\right)\left(\Pi_{1}\right)+g_{\mu \nu}\left(\mathbf{e}^{\nu}\right)(P)
\end{aligned}
$$

The $\Psi$ which are elements of the space of representation of the operators above have the general form

$$
\Psi=f(P) \oplus f_{\mu}\left(P^{\mu}\right)
$$

They are expressed as a direct sum of scalars and vectors. This corresponds in the usual D.K.P. theory to the column representation

$$
\left(\begin{array}{c}
f \\
\left(f_{1}\right) \\
\left(f_{2}\right) \\
\left(f_{3}\right) \\
\left(f_{4}\right)
\end{array}\right)
$$

where $f$ is a scalar function proportional to the projector $(\mathrm{P})$ onto the scalars (see appendix) and the $\left(f_{\mu}\right)$ are vector functions. In this case the D.K.P algebra coincides with the total matrix algebra of the $(4+1)$ dimensional space (see Appendix for more details).

We now aim to arrive at a phase-space D.K.P equation. Following [1] we first regard the free particle equation

$$
\partial_{\mu} \vec{\beta}^{\mu} \Psi=0
$$

and its adjoint

$$
\partial_{\mu} \overleftarrow{\beta}^{\mu} \Psi=0
$$

where 


$$
\vec{\beta}^{\mu} \Psi=\beta^{\mu} \Psi 1
$$

and

$$
\overleftarrow{\beta}^{\mu} \Psi=1 \Psi \beta^{\mu}
$$

We define two sets of elements in the algebra:

$$
\stackrel{+}{\beta}_{\mu}=(P)\left(e_{\mu}\right)+g_{\mu \nu}\left(e^{\nu}\right)(P)
$$

and

$$
\bar{\beta}_{\mu}=(P)\left(e_{\mu}\right)-g_{\mu \nu}\left(e^{\nu}\right)(P) .
$$

It can be verified that these are generators of two D.K.P algebras satisfying the relations

$$
\stackrel{+}{\beta}_{\mu} \stackrel{+}{\beta}_{\nu} \stackrel{+}{\beta}_{\lambda}+\stackrel{+}{\beta}_{\lambda} \stackrel{+}{\beta}_{\nu} \stackrel{+}{\beta}_{\mu}=g_{\mu \nu} \stackrel{+}{\beta}_{\lambda}+g_{\nu \lambda} \stackrel{+}{\beta}_{\mu}
$$

and

$$
\bar{\beta}_{\mu} \bar{\beta}_{\nu} \bar{\beta}_{\lambda}+\bar{\beta}_{\lambda} \bar{\beta}_{\nu} \bar{\beta}_{\mu}=-g_{\mu \nu} \bar{\beta}_{\lambda}-g_{\nu \lambda} \bar{\beta}_{\mu} .
$$

It is convenient to relate the $\hat{\beta}_{\mu}^{+}$and the $\bar{\beta}_{\mu}$ to the $\overrightarrow{\beta_{\mu}}$ and $\overleftarrow{\beta_{\mu}}$. For we define

$$
\begin{aligned}
& \vec{\eta}_{5}=\vec{\eta}_{1} \vec{\eta}_{2} \vec{\eta}_{3} \vec{\eta}_{4} \\
& \overleftarrow{\eta}_{5}=\overleftarrow{\eta}_{1} \overleftarrow{\eta}_{2} \overleftarrow{\eta}_{3} \overleftarrow{\eta}_{4}
\end{aligned}
$$

where

$$
\stackrel{\leftarrow}{\eta}_{\mu}=2\left(\overleftrightarrow{\vec{\beta}}_{\mu}\right)^{2}-1
$$

The relations we want are

$$
\stackrel{+}{\beta}_{\mu}=\vec{\beta}_{\mu} \quad \text { and } \quad \bar{\beta}_{\mu}=\omega \overleftarrow{\beta}_{\mu}
$$

where

$$
\omega=\overleftarrow{\eta}_{5} \vec{\eta}_{5}=\vec{\eta}_{5} \overleftarrow{\eta}_{5}, \quad \omega^{2}=1
$$

and it is verified that the element $\omega$ anticommutes with all the $\stackrel{\leftarrow}{\beta}_{\mu}$.

From these relations we can write

$$
b_{\mu}^{\dagger}=\frac{1}{2}\left(\stackrel{+}{\beta}_{\mu}-\bar{\beta}_{\mu}\right) ; \quad b_{\nu}=\frac{1}{2}\left(\stackrel{+}{\beta}_{\nu}+\bar{\beta}_{\nu}\right)
$$

where we have adopted the convention

$$
\begin{gathered}
(P)\left(\mathbf{e}_{\mu}\right) \longrightarrow b_{\mu} \\
g_{\mu \nu}\left(\mathbf{e}^{\nu}\right)(P) \longrightarrow b_{\mu}^{\dagger} .
\end{gathered}
$$

The construction of the above relations amounts to apply the techniques introduced in [1] to derive a Liouville type equation in the generalized phase space. The relations (20) are analogous to the relations between the Grassmann algebra and the Clifford algebra shown in [1]. Here ThoweverГthey have a different interpretation.

\section{D.K.P equation on the gen- eralized phase space}

The above algebraic calculus is based on an algebra for vectors and covectors (see Appendix). Therefore it gives rise to a structure similar to the momentum phase space i.e the image of a local chart of a cotangent bundle [8]. It should be noted that the bilinear form $B$ (see Appendix) used to construct the D.K.P algebra is not the symplectic bilinear form but a pseudo-euclidean form on phase space $\mathbf{W}$. The space $\mathbf{W}$ enters the theory of the relativistic D.K.P particle in the usual $(X, p)$ phase space in the following way: let $M$ be the phase space of continuous variables $\left(X^{\mu}, p^{\mu}\right)$ defined by the relativistic version of the Wigner-Moyal transformation [1].

$$
\mathcal{F}\left(X^{\mu}, p^{\mu}\right)=\frac{1}{2 \pi} \int V\left(x^{, \mu}, x^{\mu}\right) e^{-i p_{\mu} \xi^{\mu}} d^{4} \xi
$$

where

$$
X^{\mu}=\frac{x^{\mu}+x^{\mu}}{2}, \quad \xi^{\mu}=x^{\mu}-x^{, \mu} .
$$

The generalized relativistic phase space of the D.K.P particle can modulo relativistic constraints be viewed as the product space

$$
T \stackrel{\text { def }}{=} M \otimes D_{p}^{p+1}(\mathbf{W})
$$

where $D_{p}^{p+1}(\mathbf{W})$ is the spin space of the D.K.P particle in $\mathbf{W} . T$ is the total space. A state of the particle is represented by the Wigner function defined on $T$. The set of these states will then form the domain of the operators $\left(X, p, \frac{\partial}{\partial X}, \frac{\partial}{\partial p}, b^{\dagger \mu}, b^{\mu}\right)$ that will be involved in the Liouville equation. Next $\Gamma$ we derive this equation for the free D.K.P scalar particle. We begin with the D.K.P equation with no mass. The following steps serve to give a brief summary of the techniques used in [1] to obtain the Liouville operator. We go back to equations (8) and (9) and write it down for the density matrix $V$ in the usual x-representation: 


$$
\begin{gathered}
\frac{\partial}{\partial x^{\prime \mu}} \vec{\beta}^{\mu} V\left(x^{, \mu}, x^{\mu}\right)=0 \\
\frac{\partial}{\partial x^{\mu}} V\left(x^{, \mu}, x^{\mu}\right) \stackrel{\leftarrow}{\beta}^{\mu}=0 .
\end{gathered}
$$

$V$ is a function defined on the space $T$. The dependence on the spin indices has been dropped for convenience. Multiplying (25) by $\omega$ and using the definitions (18) we get

$$
\begin{aligned}
& \frac{\partial}{\partial x^{\prime \mu}} \stackrel{+}{\beta}^{\mu} V\left(x^{\prime \mu}, x^{\mu}\right)=0 \\
& \frac{\partial}{\partial x^{\mu}} \bar{\beta}^{\mu} V\left(x^{\prime \mu}, x^{\mu}\right)=0 .
\end{aligned}
$$

Now we use the relations (20) to write:

$$
\begin{aligned}
& \left(b^{\mu}+b^{\dagger \mu}\right) \partial_{\mu}^{\prime} V=0 \\
& \left(b^{\mu}-b^{\dagger \mu}\right) \partial_{\mu} V=0 .
\end{aligned}
$$

We change variables according to

$$
X^{\mu}=\frac{x^{, \mu}+x^{\mu}}{2} ; \quad \xi^{\mu}=x^{\mu}-x^{, \mu} .
$$

Thus (28) and (29) become

$$
\begin{aligned}
& \left(b^{\mu}+b^{\dagger \mu}\right)\left[\frac{1}{2} \frac{\partial}{\partial X^{\mu}}-\frac{\partial}{\partial \xi^{\mu}}\right] V=0 \\
& \left(b^{\mu}-b^{\dagger \mu}\right)\left[\frac{1}{2} \frac{\partial}{\partial X^{\mu}}+\frac{\partial}{\partial \xi^{\mu}}\right] V=0 .
\end{aligned}
$$

The addition and subtraction of the above equations give

$$
\begin{aligned}
& \left(\frac{1}{2} b^{\mu} \frac{\partial}{\partial X^{\mu}}-b^{\dagger \mu} \frac{\partial}{\partial \xi^{\mu}}\right) V=0 \\
& \left(\frac{1}{2} b^{\dagger \mu} \frac{\partial}{\partial X^{\mu}}-b^{\mu} \frac{\partial}{\partial \xi^{\mu}}\right) V=0 .
\end{aligned}
$$

Under the Wigner-Moyal transformation (23) these equations become

$$
\begin{aligned}
& \left(\frac{1}{2} b^{\mu} \frac{\partial}{\partial X^{\mu}}-i b^{\dagger \mu} p_{\mu}\right) F\left(X^{\mu}, p^{\mu}\right)=0 \\
& \left(\frac{1}{2} b^{\dagger \mu} \frac{\partial}{\partial X^{\mu}}-i b^{\mu} p_{\mu}\right) F\left(X^{\mu}, p^{\mu}\right)=0 .
\end{aligned}
$$

These are the massless free-particle D.K.P equations on the generalized phase space $T$. The functions $F\left(X^{\mu}, p^{\mu}\right)$ has the following module structure

$$
F=\mathcal{F} \otimes \chi, \quad \chi \in D_{p}^{p+1}(W)
$$

where $\mathcal{F}\left(X^{\mu}, p^{\mu}\right)$ is the Wigner function defined on the continuous variables only.

The square of the operator

$$
\left(\frac{1}{2} b^{\dagger \mu} \frac{\partial}{\partial X^{\mu}}-i b^{\mu} p_{\mu}\right)
$$

is the Liouville operator on $F$. In fact $\Gamma$ we have

$$
\left(\frac{1}{2} b^{\dagger \mu} \frac{\partial}{\partial X^{\mu}}-i b^{\mu} p_{\mu}\right)^{2} F=0
$$

or

$$
\begin{gathered}
\frac{1}{4} b^{\dagger \mu} b^{\dagger \nu} \frac{\partial}{\partial X^{\mu}} \frac{\partial}{\partial X^{\nu}} F-i \frac{1}{2} b^{\dagger \mu} b^{\nu} \frac{\partial}{\partial X^{\mu}} p_{\nu} F \\
-\frac{1}{2} b^{\mu} b^{\dagger \nu} p_{\mu} \frac{\partial}{\partial x^{\nu}} F-b^{\mu} b^{\nu} p_{\mu} p_{\nu} F=0 .
\end{gathered}
$$

And by using the relations

$$
\left[b^{\dagger \mu}, b^{\nu}\right]_{+}=\delta^{\mu \nu}(P)+b^{\dagger \mu} b^{\nu}
$$

$$
\left[b^{\dagger \mu}, b^{\dagger \nu}\right]_{+}=0
$$

$$
\left[b^{\mu}, b^{\nu}\right]_{+}=0
$$

where $[A, B]_{+}=A B+B A \Gamma$ we obtain

$$
\left(\delta^{\mu \nu}(P)+b^{\dagger \nu} b^{\mu}\right) p_{\nu} \frac{\partial}{\partial X^{\mu}} F=0 .
$$

This is the classical Liouville equation for a free particle. Notice that the equation has two terms. The first one accounts for the scalar part of $F$ and the second for the vector part of $F$. The operator

$$
\left[b^{\dagger \mu}, b^{\nu}\right]_{+}=\delta^{\mu \nu}(P)+b^{\dagger \mu} b^{\nu}
$$

leaves the space of states stable which makes the equation algebraicaly consistent. It is noteworth to point out that besides the scalar part $\Gamma$ there is also a term involving $b^{\dagger \mu} b^{\nu}$. This is because the D.K.P is a matrix algebra of the tensors of order $p$ and $p+1$. In the case in consideration $\Gamma$ we are taking $p=0$ which justify the term $b^{\dagger \mu} b^{\nu}$ corresponding to $p+1$. The making of the equation (39) opens the possibility of deriving the Liouville operator in a more general situation; we 
shall regard the D.K.P particle with mass $m$ in an external electromagnetic field (in units $\hbar=1$ and metric $(1 \mathrm{~F} 1 \mathrm{~F}-\mathrm{F} 1))$. The equations for $V$ are:

$$
\begin{aligned}
& \vec{\beta}^{\mu}\left(\frac{\partial}{\partial x^{\mu}}-\frac{i e}{c} A_{\mu}\left(x^{\nu}\right)\right) V+m V=0 \\
& \overleftarrow{\leftrightarrow}^{\mu}\left(\frac{\partial}{\partial x^{\mu}}+\frac{i e}{c} A_{\mu}\left(x^{\nu}\right)\right) V+m V=0 .
\end{aligned}
$$

Multiplying (42) by $\omega$ and using (18) we get:

$$
\begin{gathered}
\stackrel{+}{\beta}^{\mu}\left(\frac{\partial}{\partial x^{\mu}}-\frac{i e}{c} A_{\mu}\left(x^{, \nu}\right)\right) V+m V=0 \\
\bar{\beta}^{\mu}\left(\frac{\partial}{\partial x^{\mu}}-\frac{i e}{c} A_{\mu}\left(x^{\nu}\right)\right) V+\omega m V=0 .
\end{gathered}
$$

We now use the relations (20) to write

$$
\begin{aligned}
& \left(b^{\mu}+b^{\dagger \nu}\right)\left(\frac{\partial}{\partial x^{, \mu}}-\frac{i e}{c} A_{\mu}\left(x^{, \nu}\right)\right) V+m V=0 \\
& \left(b^{\mu}-b^{\dagger \nu}\right)\left(\frac{\partial}{\partial x^{\mu}}-\frac{i e}{c} A_{\mu}\left(x^{\nu}\right)\right) V+\omega m V=0 .
\end{aligned}
$$

Changing the variables to $X_{\mu}$ and $\xi_{\mu}$ Taccording to (30) Tand further expanding in the potentials yields

$$
A_{\mu}\left(X^{\nu} \pm \xi^{\nu} / 2\right) \simeq A_{\mu}\left(X^{\nu}\right) \pm \frac{\xi^{\alpha}}{2} \frac{\partial}{\partial X^{\alpha}} A_{\mu}\left(X^{\nu}\right)
$$

Following the same steps which led to (33) and (34) one gets

$$
\begin{aligned}
& {\left[b^{\mu}\left(\frac{\partial}{\partial X^{\mu}}+\frac{i e}{c} \xi^{\alpha} \frac{\partial}{\partial X^{\alpha}} A_{\mu}\right)-2 b^{\dagger \mu}\left(\frac{\partial}{\partial \xi^{\mu}}+\frac{i e}{c} A_{\mu}\right)+m(1-\omega)\right] V=0} \\
& {\left[b^{\dagger \mu}\left(\frac{\partial}{\partial X^{\mu}}+\frac{i e}{c} \xi^{\alpha} \frac{\partial}{\partial X^{\alpha}} A_{\mu}\right)-2 b^{\mu}\left(\frac{\partial}{\partial \xi^{\mu}}+\frac{i e}{c} A_{\mu}\right)+m(1+\omega)\right] V=0}
\end{aligned}
$$

which under the Wigner-Moyal transformation become

$$
\begin{gathered}
{\left[b^{\mu}\left(\frac{\partial}{\partial X^{\mu}}+\frac{e}{c} \xi^{\alpha} \frac{\partial}{\partial X^{\alpha}} A_{\mu} \frac{\partial}{\partial p_{\alpha}}\right)-2 i b^{\dagger \mu}\left(p_{\mu}-\frac{e}{c} A_{\mu}\right)+m(1-\omega)\right] F=0} \\
{\left[b^{\dagger \mu}\left(\frac{\partial}{\partial X^{\mu}}+\frac{e}{c} \xi^{\alpha} \frac{\partial}{\partial X^{\alpha}} A_{\mu} \frac{\partial}{\partial p_{\alpha}}\right)-2 i b^{\mu}\left(p_{\mu}-\frac{e}{c} A_{\mu}\right)+m(1+\omega)\right] F=0 .}
\end{gathered}
$$

These are the D.K.P equations in the generalized phase space. The corresponding Liouville operator is the square of the operator on $F$ given in (47). The square of(48) leads to the same equation. In order to compute the square of the above operator we first rewrite (48) taking into account the fact that $\omega^{2}=1$. Thus $\Gamma$

$$
\left(-\frac{b^{\dagger \mu}}{2} \frac{\partial}{\partial X^{\mu}}+\frac{e}{2 c} b^{\dagger \mu} \frac{\partial A_{\mu}}{\partial X^{\alpha}} \frac{\partial}{\partial p_{\alpha}}+i b^{\mu}\left(p_{\mu}-\frac{e}{c} A_{\mu}\right)\right) F=m F
$$

This equation is an eigenvalue equation for $F$. The Liouville operator $L_{D K P}$ is the square of the operator

$$
-\frac{b^{\dagger \mu}}{2} \frac{\partial}{\partial X^{\mu}}+\frac{e}{2 c} b^{\dagger \mu} \frac{\partial A_{\mu}}{\partial X^{\alpha}} \frac{\partial}{\partial p_{\alpha}}+i b^{\mu}\left(p_{\mu}-\frac{e}{c} A_{\mu}\right)
$$

with $m^{2}$ as the eigenvalue. In order to compute the square we define the following notation

$$
L^{[\mu]}:=-\frac{b^{\dagger \mu}}{2} \frac{\partial}{\partial X^{\mu}}+\frac{e}{2 c} b^{\dagger \mu} \frac{\partial A_{\mu}}{\partial X^{\alpha}} \frac{\partial}{\partial p_{\alpha}}+i b^{\mu}\left(p_{\mu}-\frac{e}{c} A_{\mu}\right) .
$$

Now we compute

$$
\left[\frac{L^{[\mu]} L^{[\nu]}+L^{[\nu]} L^{[\mu]}}{2}\right] F=m^{2} F
$$


which gives

$$
\begin{aligned}
L_{D K P}= & \left((P) \delta^{\mu \nu}+b^{\dagger \nu} b^{\mu}\right)\left(p_{\mu}-\frac{e}{c} A_{\mu}\right) \frac{\partial}{\partial X^{\nu}} \\
& -\frac{e}{2 c}\left(b^{\dagger \nu} b^{\mu}-b^{\dagger \mu} b^{\nu}\right)\left(\frac{\partial A_{\mu}}{\partial X^{\nu}}-\frac{\partial A_{\nu}}{\partial X^{\mu}}\right) \\
& +\frac{e}{c}\left(b^{\dagger \nu} b^{\mu}+\delta^{\mu \nu}(P)\right)\left(p_{\mu}-\frac{e}{c} A_{\mu}\right) \frac{\partial A_{\nu}}{\partial X^{\alpha}} \frac{\partial}{\partial p_{\alpha}}-2 i m^{2} .
\end{aligned}
$$

Clearly one can distinguish two terms. Firstly $\Gamma$ the scalar part $\Gamma i . e \Gamma$

$$
L_{D K P}^{s}=(P) \delta^{\mu \nu}\left(p_{\mu}-\frac{e}{c} A_{\mu}\right) \frac{\partial}{\partial X^{\nu}}+\frac{e}{c}(P) \delta^{\mu \nu}\left(p_{\mu}-\frac{e}{c} A_{\mu}\right) \frac{\partial A_{\nu}}{\partial X^{\alpha}} \frac{\partial}{\partial p_{\alpha}}
$$

and secondly

$$
\begin{aligned}
L_{D K P}^{v}= & b^{\dagger \nu} b^{\mu}\left(p_{\mu}-\frac{e}{c} A_{\mu}\right) \frac{\partial}{\partial X^{\nu}}+\frac{e}{c} b^{\dagger \nu} b^{\mu}\left(p_{\mu}-\frac{e}{c} A_{\mu}\right) \frac{\partial A_{\nu}}{\partial X^{\alpha}} \frac{\partial}{\partial p_{\alpha}} \\
& -\frac{e}{2 c}\left(b^{\dagger \nu} b^{\mu}-b^{\dagger \mu} b^{\nu}\right)\left(\frac{\partial A_{\mu}}{\partial X^{\nu}}-\frac{\partial A_{\nu}}{\partial X^{\mu}}\right) .
\end{aligned}
$$

The total Liouville operator is $L_{D K P}^{s}+L_{D K P}^{v}-$ $2 \mathrm{im}^{2}$. Due to the presence of the projector $\Pi_{0} \equiv P$ (see Appendix) in (51) $\Gamma L_{D K P}^{s}$ operates only on the scalar part of $F$. We can leave $P$ out of $L_{D K P}^{s}$ since it is just a unit on its range. Thus (51) can be read without $P$. Hence $L_{D K P}^{s}$ is just the Liouville operator for the Klein-Gordon equation with Euclidean metric $\delta^{\mu \nu}$ [3]. The two terms in $L_{D K P}^{s}$ represent: the modified contribution to $L_{D K P}$ coming from the motion of the trajectory in phase space and the electromagnetic force respectively.

In (53) we have the vector part of $F$ being modified by two similar terms. Contrary to $(51) \Gamma$ these terms operate only on the vector part since they vanish on the scalar part. The third term shows the effect of the spin of the DKP particle which appears coupled with the electromagnetic field only in the vector part. Here there is a complete similarity with the Liouville operator for the Dirac equation derived in [1]. The term $\left(b^{\dagger \nu} b^{\mu}-b^{\dagger \mu} b^{\nu}\right)$ is just the spin operator $S_{\mu \nu}$ exhibited in [9]. Notice that despite we are analyzing the scalar case $(p=0) \Gamma$ there is always the $p+1$ term involved. As aforementioned $\Gamma$ this is because the D.K.P algebra gives rise to a total matrix algebra of order $p$ and $p+1$.

\section{Conclusions}

We have shown how to apply the phase space approach proposed by Bohm and Hiley [1] in order to find a formalism describing bosonic particles. In such an approach the physical property of spin appears in a classical relativistic algebraic formalism in phase space. This space can be viewed as a product space which naturally embodies new kind of algebraic operators besides the usual differential operators that feature in the plain Liouville formalism. The generators of the D.K.P algebras are constructed in the standard fashion used for deriving Clifford algebras out of bilinear forms.

Via a relativistic version of the Wigner-Moyal transformation we have arrived at two Liouville type equations. One for free particles and the other for particles in the presence of an electromagnetic field. In previous works on this subject Bohm and Hiley studied the Dirac equation and its non-relativistic case [10]. Therefore our results add to the program since we were able to exhibt an algebraic development for the relativistic phase space describing bosons as well. As a consequence $\Gamma$ the interpretation of the solutions of the Liouville equation as spin fields having certain independence of motion $\Gamma$ and the phase trajectories as whole depending on the 
interaction of these fields with the electromagnetic field has also been verified for bosonic systems.

So far we have obtained the Liouville equation for D.K.P particles corresponding to $p=0$ in the D.K.P algebra. However it is possible to generalize this result for any allowed values of the label $p$. A further work in this direction is in progress and should be published in a forthcoming paper.

\section{Appendix}

\section{V-I The extended Grassmann algebra of a phase space}

Let $\mathbf{V}$ be a n-dimensional vector space and $\mathbf{V}^{*}$ its dual space. A phase space $\mathbf{W}$ is defined as

$$
\mathbf{W}=\mathbf{V} \oplus \mathbf{V}^{*}=\left\{(v, u), v \in \mathbf{V}, u \in \mathbf{V}^{*}\right\}
$$

Let $B$ be a bilinear form defined on WГi.e. $B$ : $\mathbf{W} \times \mathbf{W} \longrightarrow R$ or $C \quad$ (Real or Complex field) given by

$$
2 B\left((v, u),\left(v^{\prime}, u^{\prime}\right)\right)=\left\langle u, v^{\prime}\right\rangle+\left\langle u^{\prime}, v\right\rangle
$$

where $\langle\cdot, \cdot\rangle$ is the natural pairing of vectors and covectors. This bilinear form is non-singular and establishes an isomorphism between $\mathbf{W}$ and $\mathbf{W}^{*}$; the choice of this bilinear form on $\mathbf{W}$ embeds it into a Clifford algebra but in this case a Clifford algebra of the phase space W.

The standard way to construct the algebra from $B$ is as follows: Let $\mathbf{T}(\mathbf{W})$ be the tensor algebra over $\mathbf{W}$ i.e.

$$
\mathbf{T}(\mathbf{W})=\bigoplus_{i=0}^{\infty} \mathbf{T}^{(i)}(\mathbf{W})
$$

where $\mathbf{T}^{(i)}=\mathbf{W} \otimes \mathbf{W} \otimes \cdots \otimes \mathbf{W}$ (i times) and $\mathbf{W}$ is identified with the 1-piece $\mathbf{T}^{(1)}(\mathbf{W})$ of $\mathbf{T}(\mathbf{W})$.

From the universal property of $\mathbf{T}(\mathbf{W})$ one has

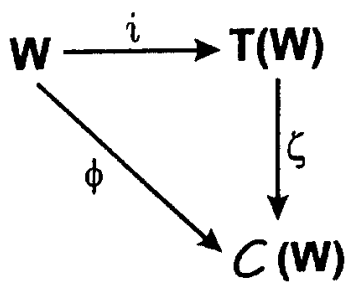

where $C(\mathbf{W})$ is an associative algebra. Let $\mathbf{I}$ be the two-sided ideal

$$
w \otimes w^{\prime}+w^{\prime} \otimes w-2 B\left(w, w^{\prime}\right) 1_{T(W)} .
$$

in $\mathbf{T}(\mathbf{W})$. Define $\mathcal{C}(\mathbf{W})=\mathbf{T}(\mathbf{W}) / \mathbf{I}$. Therefore one has

$$
\phi\left(w \otimes w^{\prime}+w^{\prime} \otimes w-2 B\left(w, w^{\prime}\right) 1_{T(W)}\right)=0,
$$

since $\phi$ is an homomorphism. This implies that

$$
\left[\phi(w), \phi\left(w^{\prime}\right)\right]_{+}=2 B\left(w, w^{\prime}\right) 1_{\mathcal{C}(\mathbf{w})}
$$

Writing $w$ as $w=v \oplus u \Gamma v \in \mathbf{V}$ and $u \in \mathbf{V}^{*}$ one gets the extended Grassmann algebra of vectors and covectors

$$
\begin{gathered}
{\left[\phi(u), \phi\left(u^{\prime}\right)\right]_{+}=\left[\phi(v), \phi\left(v^{\prime}\right)\right]_{+}=0} \\
{[\phi(v), \phi(u)]_{+}=\langle u, v\rangle 1_{\mathcal{C}(\mathbf{w})}}
\end{gathered}
$$

where one may have the identifications $\phi(v)=v \Gamma$ $\phi(u)=u$.

Writing $v$ and $u$ in a basis i.e.

$$
v=v^{i} \mathbf{e}_{i}, \quad u=u_{i} \mathbf{e}^{i}
$$

and using linearity of $\phi$ one gets

$$
\begin{gathered}
{\left[\mathbf{e}_{i}, \mathbf{e}^{j}\right]_{+}=\delta_{i}^{j} 1_{\mathcal{C}(\mathbf{w})}} \\
{\left[\mathbf{e}_{i}, \mathbf{e}_{j}\right]_{+}=\left[\mathbf{e}^{i}, \mathbf{e}^{j}\right]_{+}=0}
\end{gathered}
$$

Therefore $\mathcal{C}(\mathbf{W})$ is an algebra generated by vectors and covectors with basis comprised by the $2^{2 n}$ elements

$$
\left(\mathbf{e}_{\mathbf{1}}\right)^{r_{1}} \cdots\left(\mathbf{e}_{\mathbf{n}}\right)^{r_{n}}\left(\mathbf{e}^{\mathbf{n}}\right)^{s_{n}} \cdots\left(\mathbf{e}^{\mathbf{1}}\right)^{s_{1}} ; \quad r, s=0,1
$$

In order to build up a canonical basis for $\mathcal{C}(\mathbf{W})$ one defines the following primitive idempotent

$$
P=N_{1} \cdots N_{n} ; \quad n=\operatorname{dim} \mathbf{V}
$$

and

$$
N_{j}=\mathbf{e}_{j} \mathbf{e}^{j}, \quad \text { no sum. }
$$

It is easily verified that $P^{2}=P$. The canonical basis for $\mathcal{C}(\mathbf{W})$ is written as

$$
P_{j_{1} \cdots j_{p}}^{k_{1} \cdots k_{q}}=\mathbf{e}^{k_{1}} \cdots \mathbf{e}^{k_{q}} P \mathbf{e}_{j_{p}} \cdots \mathbf{e}_{j_{1}}
$$

which implies 


$$
\left(P_{j_{1} \cdots j_{p}}^{k_{1} \cdots k_{q}}\right)\left(P_{h_{1} \cdots h_{p}}^{i_{1} \cdots i_{q}}\right)=\delta_{p, q} \delta_{j_{1} \cdots j_{p}}^{i_{1} \cdots i_{q}}\left(P_{h_{1} \cdots h_{p}}^{k_{1} \cdots k_{q}}\right)
$$

Hence these $2^{2 n}$ elements are linearly independents and a general element of $\mathcal{C}(\mathbf{W})$ can be expressed as a linear combination

$$
\Gamma=\sum_{p, q=0}^{n}(p ! q !)^{-1} A_{k_{1} \cdots k_{q}}^{j_{1} \cdots j_{p}} P_{j_{1} \cdots j_{p}}^{k_{1} \cdots k_{q}}
$$

or

$$
\Gamma=\sum_{p, q=0}^{n}(p ! q !)^{-1} A_{\mathbf{K}_{q}}^{\mathbf{J}_{p}} P_{\mathbf{J}_{p}}^{\mathbf{K}_{q}}
$$

where we have adopted the multi-index notation

$$
\begin{gathered}
\mathbf{J}_{p}=j_{1} \cdots j_{p} \\
\mathbf{K}_{q}=k_{1} \cdots k_{q}
\end{gathered}
$$

and sum in repeated indices is also understood.

\section{V-II The spinor spaces of $\mathcal{C}(\mathbf{W})$}

We shall now consider the regular representation of $\mathcal{C}(\mathbf{W})$ [11]. The regular representaion of an algebra is a representation of the algebra on itself. An irreducible representation of $\mathcal{C}(\mathbf{W})$ can be constructed on its minimum left ideals [7]. According to Cartan [12] $\Gamma$ the minimum left ideal of the Clifford algebra can be taken as the space of spinors of the algebra. The group of innerautomorphisms of the algebra acts irreducibly on the minimum left ideals thus yielding a spinorial representation. A way to construct minimum left ideals in an algebra is to use a primitive idempotent. In our case the element $P$ introduced in (61) happens to be a primitive idempotent[13] since $\Gamma$

$$
P^{2}=P, P^{*}=P, \quad P \Gamma P=c P ; \quad \Gamma \in \mathcal{C}(\mathbf{W}), c \in C .
$$

Hence a minimum left ideal of $\mathcal{C}(\mathbf{W})$ can be formed by the projection

\section{$\Gamma P$}

for every $\Gamma \in \mathcal{C}(\mathbf{W})$. We denote the projected space by $\mathcal{C}_{\mathcal{P}}(\mathbf{W})$.

Analogously one can form the space $P \Gamma$ denoted by $\mathcal{C}^{\mathcal{P}}(\mathbf{W}) \Gamma$ which is a minimun right ideal of $\mathcal{C}(\mathbf{W})$. It turns out that $\mathcal{C}(\mathbf{W})$ is a matrix algebra and has a representation on the subspaces $\mathcal{C}_{\mathcal{P}}(\mathbf{W})$ and $\mathcal{C}^{\mathcal{P}}(\mathbf{W})$. Let us see how this result works in terms of components. For take

$$
\Gamma=\sum_{p, q=0}^{n}(p ! q !)^{-1} A_{\mathbf{K}_{q}}^{\mathbf{J}_{p}} P_{\mathbf{J}_{p}}^{\mathbf{K}_{q}}
$$

and project it down into $\mathcal{C}_{\mathcal{P}}(\mathbf{W})$,i.e

$$
\Gamma P=\sum_{p, q=0}^{n}(p ! q !)^{-1} A_{\mathbf{K}_{q}}^{\mathbf{J}_{p}} P_{\mathbf{J}_{p}}^{\mathbf{K}_{q}}(P)
$$

By taking into account the relations $\mathbf{e}_{i}(P)=$ $(P) \mathbf{e}^{j}=0$ as well as rules (62) we get

$$
\begin{gathered}
\Psi:=\Gamma P=\sum_{q=0}^{n}(q !)^{-1} A_{\mathbf{K}_{q}} P^{\mathbf{K}_{q}}, \\
\Psi \in \mathcal{C}_{\mathcal{P}}(\mathbf{W}) .
\end{gathered}
$$

Now the space $C_{P}(\mathbf{W})$ of elements $\Psi$ plays the role of the space of representations of $\mathcal{C}(\mathbf{W})$. Indeed it can be easily verified that

$$
\begin{gathered}
\Gamma: \Psi \longrightarrow \Psi^{\prime} \\
\Gamma \Psi=\Psi^{\prime} ; \quad \Gamma \in \mathcal{C}(\mathbf{W}) ; \quad \Psi, \Psi^{\prime} \in \mathcal{C}_{\mathcal{P}}(\mathbf{W}) .
\end{gathered}
$$

\section{V-III The Dirac algebra}

The algebra $\mathcal{C}(\mathbf{W})$ developed above is an affine algebra since it is generated by a set of vectors and covectors defined independently. When a metric is available one can distinguish two metric subalgebras in $\mathcal{C}(\mathbf{W})$ generated by

$$
\gamma_{\mu}^{( \pm)}=\mathbf{e}_{\mu} \pm g_{\mu \nu} \mathbf{e}^{\nu}
$$

one for each sign of the metric.

Using relations (60) it follows that

$$
\begin{gathered}
{\left[\gamma_{\mu}^{( \pm)}, \gamma_{\nu}^{( \pm)}\right]_{+}= \pm 2 g_{\mu \nu} 1_{\mathcal{C}(\mathcal{W})}} \\
{\left[\gamma_{\mu}^{(+)}, \gamma_{\nu}^{(-)}\right]_{+}=0}
\end{gathered}
$$

Here an important point on the notation is in order[2]. The element noted by $g_{\mu \nu} \mathbf{e}^{\nu}$ does not mean to lower the indices $\nu$ with the metric. We just mean that there is a sum on $\nu$ thus leaving free the indice $\mu$ to 
balance the equality. In this regard we should point out that the multiplication rules of the algebra depend on the covariant or contravariant nature of the generators.

The elements $\gamma_{\mu}^{+}$generates a Clifford algebra corresponding to the positive metric $g_{\mu \nu}$. When $n=4$ and $g_{\mu \nu}$ is the Minkowski metric $\Gamma$ we have the Dirac algebra of space-time.

\section{V-IV The Duffin-Kemmer-Petiau algebras}

The elements

$$
\Pi_{p}=(p !)^{-1} P_{\mathbf{J}_{P}}^{\mathbf{J}_{P}}
$$

are idempotent elements of $\mathcal{C}(\mathbf{W})$. The unity of the algebra has an idempotent decomposition

$$
1_{\mathcal{C}(\mathbf{W})}=\sum_{p=0}^{n} \Pi_{p} ; \quad \Pi_{0}:=P
$$

The elements $\Pi_{p}$ satisfy some important properties $\Gamma$ namely $\Gamma$

$$
\begin{gathered}
\left(\Pi_{p}\right)\left(\Pi_{q}\right)=\delta_{p, q}\left(\Pi_{p}\right) \\
\left(\mathbf{e}^{j}\right)\left(\Pi_{p}\right)=\left(\Pi_{p+1}\right)\left(\mathbf{e}^{j}\right) \\
\left(\Pi_{p}\right)\left(\mathbf{e}_{j}\right)=\left(\mathbf{e}_{j}\right)\left(\Pi_{p+1}\right)
\end{gathered}
$$

The generators of the D.K.P algebra which is denoted by $\beta_{\mu}^{(p)}$ satisfy the following fundamental relations:

$$
\beta_{\mu}^{(p)} \beta_{\nu}^{(p)} \beta_{\gamma}^{(p)}+\beta_{\gamma}^{(p)} \beta_{\nu}^{(p)} \beta_{\mu}^{(p)}=g_{\mu \nu} \beta_{\gamma}^{(p)}+g_{\gamma \nu} \beta_{\mu}^{(p)}
$$

These generators have an expression within $\mathcal{C}(\mathbf{W})$ as follows

$$
\begin{aligned}
\beta_{j}^{(p)} & =\left(\Pi_{p}\right)\left(\mathbf{e}_{j}\right)+g_{j i}\left(\mathbf{e}^{i}\right)\left(\Pi_{p}\right) \\
& =\left(\Pi_{p}\right)\left(\mathbf{e}_{j}\right)+g_{j i}\left(\Pi_{p+1}\right)\left(\mathbf{e}^{i}\right) \\
& =\left(\mathbf{e}_{j}\right)\left(\Pi_{p+1}\right)+g_{j i}\left(\mathbf{e}^{i}\right)\left(\Pi_{p}\right)
\end{aligned}
$$

where " $\mathrm{p}$ " is the order of the linear space into which $\Pi_{p}$ projects the $\Psi \in \mathcal{C}(\mathbf{W})$.

The element $\left(\Pi_{p}\right)+\left(\Pi_{p+1}\right)$ is idempotent and commutes with all the $\beta_{j}^{(p)}$. Thus $\left(\Pi_{p}\right)+\left(\Pi_{p+1}\right)$ is the unity of the D.K.P algebra.
Despite one has a metric in the defining relations (67) T the D.K.P algebra is not always a metric subalgebra of $\mathcal{C}(\mathbf{W})$. In fact Tone can introduce the affine algebra $D_{n, p}[2]$ generated by

$$
\left(\Pi_{p}\right)\left(\mathbf{e}_{j}\right) ; \quad\left(\mathbf{e}^{j}\right)\left(\Pi_{p}\right) \quad \text { and } \quad\left(\boldsymbol{\Pi}_{\mathbf{p}+\mathbf{1}}\right)+\left(\boldsymbol{\Pi}_{\mathbf{p}}\right)
$$

It is clear that $D_{n, p}$ contains D.K.P as a subalgebra. In fact $\Gamma D_{n, p}$ coincides with D.K.P algebra when $p \neq \frac{n-1}{2}[2]$. In this case TD.K.P is an affine algebra even if the components of the $\beta_{j}^{(p)}$ are defined in terms of the components of the metric tensor. As far as $\mathcal{C}(\mathbf{W})$ is concerned the algebra $D_{n, p}$ is the total matrix algebra of the direct sum of the linear subspaces of $\mathcal{C}_{\mathcal{P}}(\mathbf{W})$ corresponding to covariant antisymmetric tensors of order "p" and "p+1". This total matrix algebra is the D.K.P algebra when $p \neq \frac{n-1}{2}$. We shall denote the space of matrices of order $p$ and $p+1$ by $D_{p}^{p+1}(\mathbf{W})$

\section{References}

[1] D. Bohm and B.J. Hiley, in "Old and New Questions in Physics, Cosmology, Philosophy, and Theoretical Biology", A. van der Merwe, ed. (Plenum, New York, 1983), p.67.

[2] M. Schönberg, Anais Acad. Bras. Cienc. 28, 11(1956), 473 (1957); 30, 1, 117, 259, 429 (1958); Suppl. Nuovo Cimento (X) 6, 356 (1957).

[3] P.R. Holand, Found. Phys. 16, 701(1986)

[4] Y. Nedjadi and R. C. Barrett, J. Phys. G.: Nucl. Part. Phys. 19, 87 (1993)

[5] O. A. Sánchez-Valenzuela and R. E. Zuazua-Vega, J. Phys. A: Math. Gen. 23, 4967 (1993)

[6] Y. S. Kim and M. E. Noz, "Phase Space Picture of Quantum Mechanics", World Scientific, London (1991)

[7] M. Riesz, in Comptes Rendus 12me Cong. Math. Scand. (Lund, 1953), p. 241

[8] L. H. Loomis and S. Sternberg, "Advanced Calculus", Addison-Wesley, Reading, Mass. (1968).

[9] N. Kemmer, Proc. R. Soc. A 173, 91 (1939)

[10] D. Bohm and B.J. Hiley, Found. Phys. 11, 179(1981).

[11] R. Hermann," Spinors, Clifford and Cayley Algebras", Interdisciplinary Mathematics Vol. VII. Math. Sci. Press. USA (1974)

[12] E. Cartan, "The Theory of Spinors", Dover Publications, New York (1966)

[13] M. C. B. Fernandes, "Geometric Algebras and the Foundations of Quantum Theory", Ph.D. Thesis, Birkbeck College, Dept. Phys., London University (1995) 